\title{
FUNDACIÓN BIODIVERSIDAD。 UNA FUNDACIÓN QUE AVANZA, QUE APUESTA, QUE IMPULSA
}

\section{Beatriz Cursach}

biodiversidad@fundacion-biodiversidad.es

www.fundacion-biodiversidad.es

Formación, investigación y cooperación son los objetivos por los que apuesta la Fundación Biodiversidad. Para llevarlos a cabo impulsa la conservación y la sensibilización ambiental, apuesta por la formación como medio para avanzar en la instauración de una nueva cultura ambiental sostenible y avanza hacia la gestión respetuosa de recursos, fomentando las iniciativas con las instituciones, los colectivos involucrados en la preservación y la conservación de la naturaleza y con el sector privado.

España cuenta con una riqueza de biodiversidad inigualable dentro de Europa, tanto en flora y fauna, como en hábitats. Hoy en día, se están desarrollando multitud de iniciativas que demuestran que la necesidad de proteger el entorno se está incorporando cada vez más a nuestra cultura, aunque no cabe duda de que el esfuerzo de todos debe ser mayor. Una de estas iniciativas fue la creación de la Fundación Biodiversidad.

Tras la firma del Convenio sobre Diversidad Biológica, en 1993, España se compromete a elaborar una Estrategia para la Conservación y el Uso Sostenible de la Biodiversidad. Así, el 23 de noviembre de 1998, el Ministerio de Medio Ambiente funda la Fundación Biodiversidad para desarrollar los objetivos de formación, investigación y cooperación en colaboración con todas aquellas entidades públicas y privadas, nacionales o internacionales, que tengan fines análogos.

La Fundación Biodiversidad se estructura a través de un Patronato entre cuyos miembros permanentes se encuentran el Ministerio de Medio Am- biente, la Agencia Española de Cooperación Internacional y el Organismo Autónomo Parques Nacionales.

La sede de la Fundación Biodiversidad se ubica en Madrid, pero para llevar a cabo sus objetivos, cuenta con dos delegaciones: Área Atlántica, en Sevilla y Mediterránea, en Palma de Mallorca. Desde Sevilla se gestionan temas relacionados con Iberoamérica, el Norte de África y el área meridional, así como los relacionados con la conservación de la Naturaleza. Desde Palma de Mallorca se impulsan, fundamentalmente, cuestiones englobadas en el ámbito Mediterráneo, Turismo sostenible y los aspectos relacionados con la calidad ambiental.

\section{COMPROMETIDA CON LA BIODI- VERSIDAD}

Para llevar a cabo su compromiso con la diversidad biológica, la Fundación Biodiversidad pretende ser foro de investigación y centro de referencia para el desarrollo y la difusión de temas rela- 
cionados con la conservación y el uso sostenible de la biodiversidad. Asimismo, aspira a contribuir a la interrelación del hombre con el medio natural y promover la relación e integración entre la cultura y la naturaleza.

La Fundación Biodiversidad trabaja para promover el desarrollo de iniciativas para la conservación de los ecosistemas y los hábitats; para divulgar la cultura del desarrollo sostenible y propiciar la cooperación internacional para lograr la preservación de las comunidades indígenas y sus entornos. Pretende también impulsar iniciativas que favorezcan los aspectos culturales, científicos, sociales, ambientales, histórico-artístico, antropológicos, etnológicos, sociológicos, económicos, jurídicos y de todos aquellos relacionados con la biodiversidad, la conservación y el uso sostenible de los recursos naturales.

La Fundación Biodiversidad busca dar respuesta a la preocupación social creciente frente a la pérdida progresiva de la diversidad biológica. El desarrollo sostenible persigue alcanzar el equilibrio entre el desarrollo económico y social con la preservación de los recursos naturales para salvaguardar la biodiversidad de nuestro país y mejorar la calidad de vida de sus ciudadanos.

Además, promueve y organiza conferencias, coloquios, jornadas, exposiciones, congresos, debates, seminarios, cursos de especialización —universitaria y de postgrado- y encuentros. Impulsa, también, campañas de sensibilización cultural y de educación ambiental, conducentes a la mentalización y formación de la sociedad en general.

\section{CONCIENCIAR A TRAVÉS DE LA FOR- MACIÓN}

La Fundación Biodiversidad cuenta con un Área de Formación, que se ocupa de la investigación, la información y la educación ambiental y entre sus propósitos se encuentra el desarrollo de un plan de actividades que incluye la realización de cursos, seminarios y conferencias, en colaboración con universidades nacionales e internacionales y la elaboración de estudios para detectar las necesidades formativas.

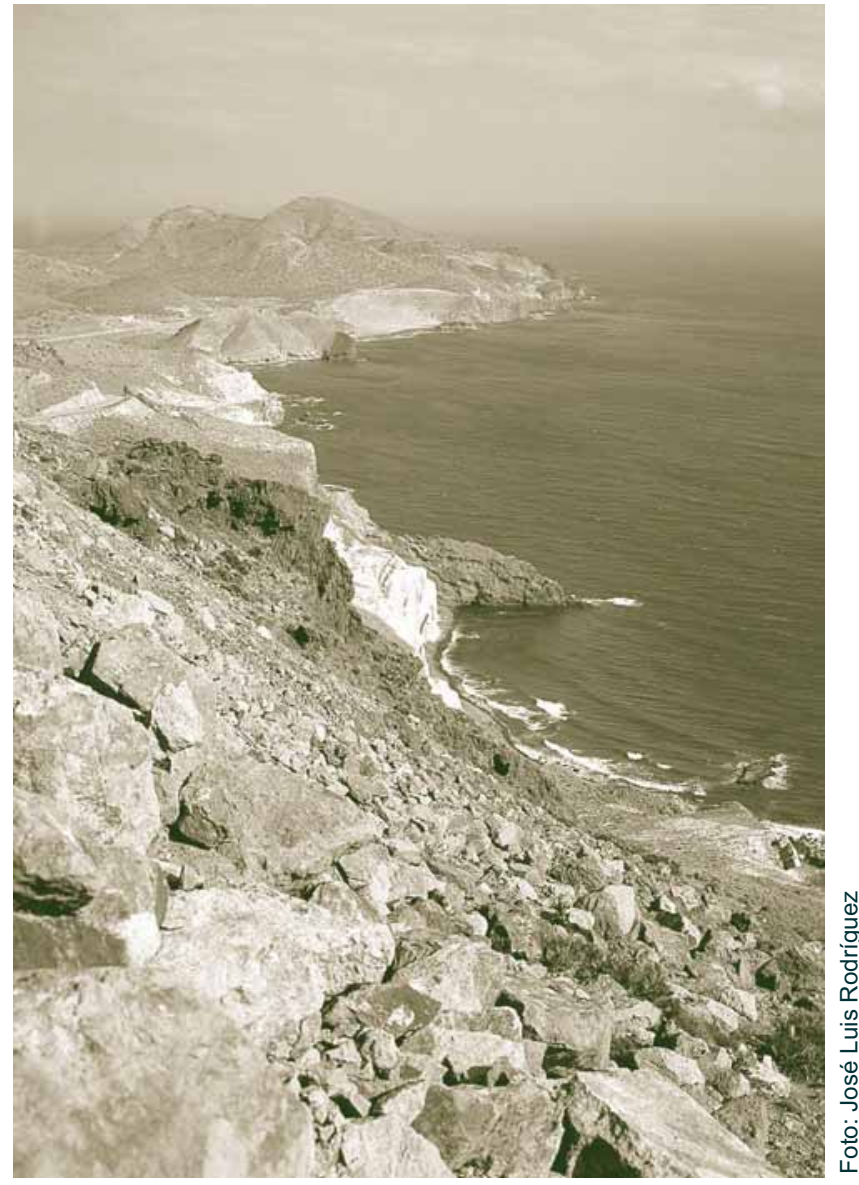

Panorámica de la costa de Cabo de Gata (Almería).

El Área de Formación contempla varias actividades formativas, entre ellas, el Master en Gestión y Administración Ambiental, cuya principal finalidad es conseguir una adecuada formación en materia ambiental destinada a los profesionales relacionados con el sector empresarial y post-universitario. A través de una metodología actual y dinámica el master se fija como objetivo formar a una nueva generación de gestores ambientales con unos conocimientos sólidos y reconocidos a nivel europeo.

La Fundación Biodiversidad organiza asimismo cursos y masters de postgrado para promover la formación académica en niveles superiores para licenciados y profesionales de diferentes sectores que quieren ampliar sus conocimientos en el campo de la biodiversidad y el desarrollo sostenible de los recursos naturales. Además, gestiona el Programa Iniciativa Empresarial y Gestión Continua del Fondo Social Europeo para PYME. La Fundación 


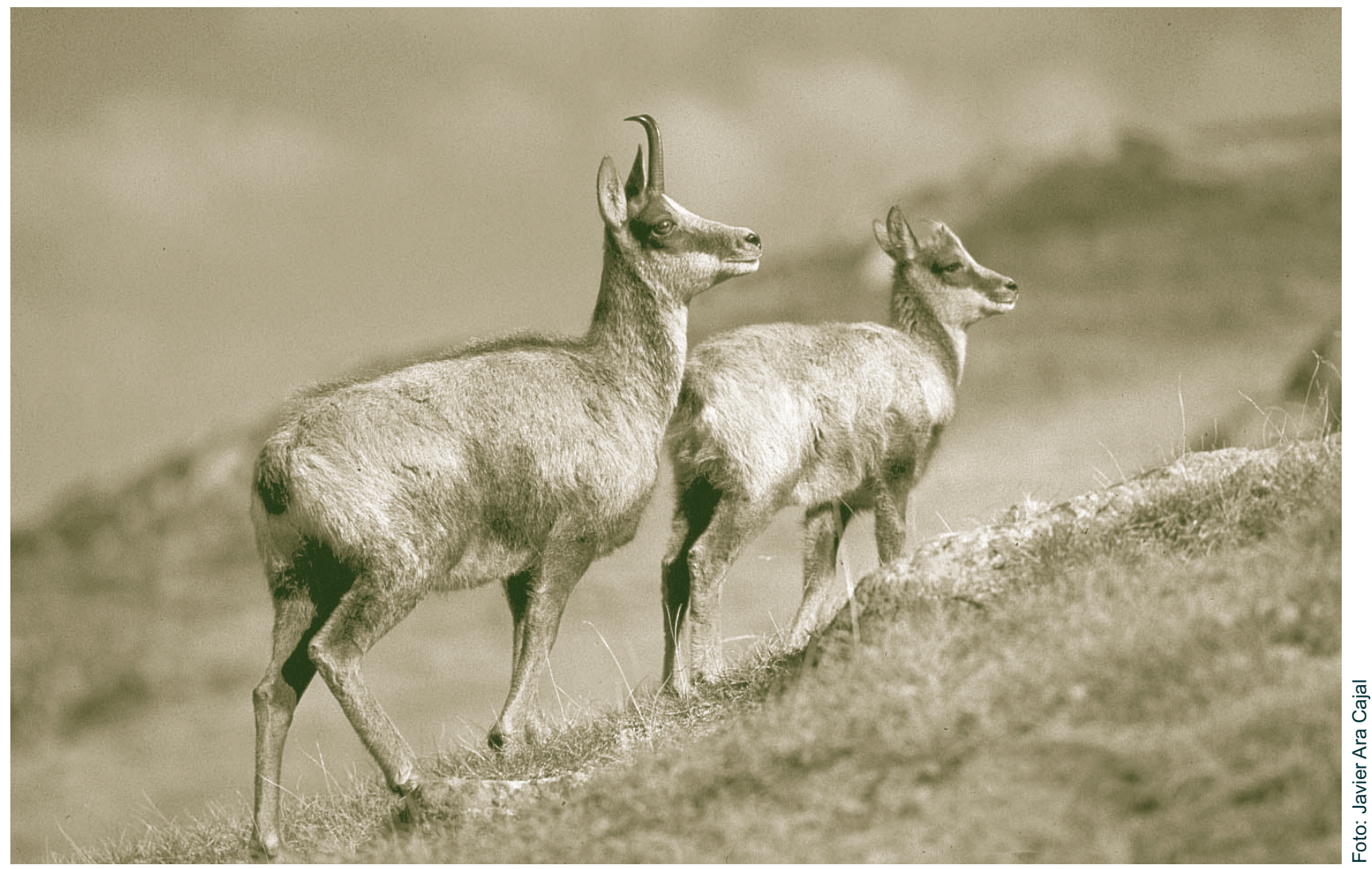

Dos ejemplares de sarrio subiendo por una ladera.

Biodiversidad coordinará el programa de acciones formativas financiadas por el Fondo Social Europeo entre los años 2001-2006.

\section{BIODIVERSIDAD SIN FRONTERAS}

La Fundación Biodiversidad desarrolla importantes convenios de cooperación con otros organismos, fundaciones e instituciones internacionales con fines comunes. En este ámbito se enmarca el programa Araucaria. Este programa es fruto de los compromisos internacionales acordados por España a partir de la Cumbre de la Tierra de 1992. Su marco de actuación es Iberoamérica y su fin es apoyar el desarrollo de proyectos y actividades destinados a la conservación de la biodiversidad y a la gestión de áreas protegidas, incluyendo programas específicos de colaboración continua.

En el programa Araucaria participan el Ministerio de Medio Ambiente, el Ministerio de Economía, el Ministerio de Hacienda, el Ministerio de
Educación y Cultura y la Agencia Española de Cooperación Internacional.

Los recursos naturales en Iberoamérica son, en la actualidad, objeto de una sobreexplotación que amenaza la sostenibilidad de los países de esta área. El programa Araucaria incide en la puesta en marcha de modelos de desarrollo sostenible que permitan frenar las pérdidas en la biodiversidad, una de las más ricas del planeta, cuyo patrimonio es de obligada protección. Las actuaciones del Programa Araucaria se dirigen hacia la actuación en la conservación de la biodiversidad y de los principales ecosistemas de Iberoamérica, así como al desarrollo humano de los pobladores locales, a la generación de un impacto real sobre la conservación de la biodiversidad in situ, que sea apreciada por las comunidades locales así como una aportación real a su desarrollo, y a contribuir al cumplimiento de las obligaciones contraídas por España en los foros internacionales en materia de cooperación para la conservación de la biodiversidad y el desarrollo sostenible. 


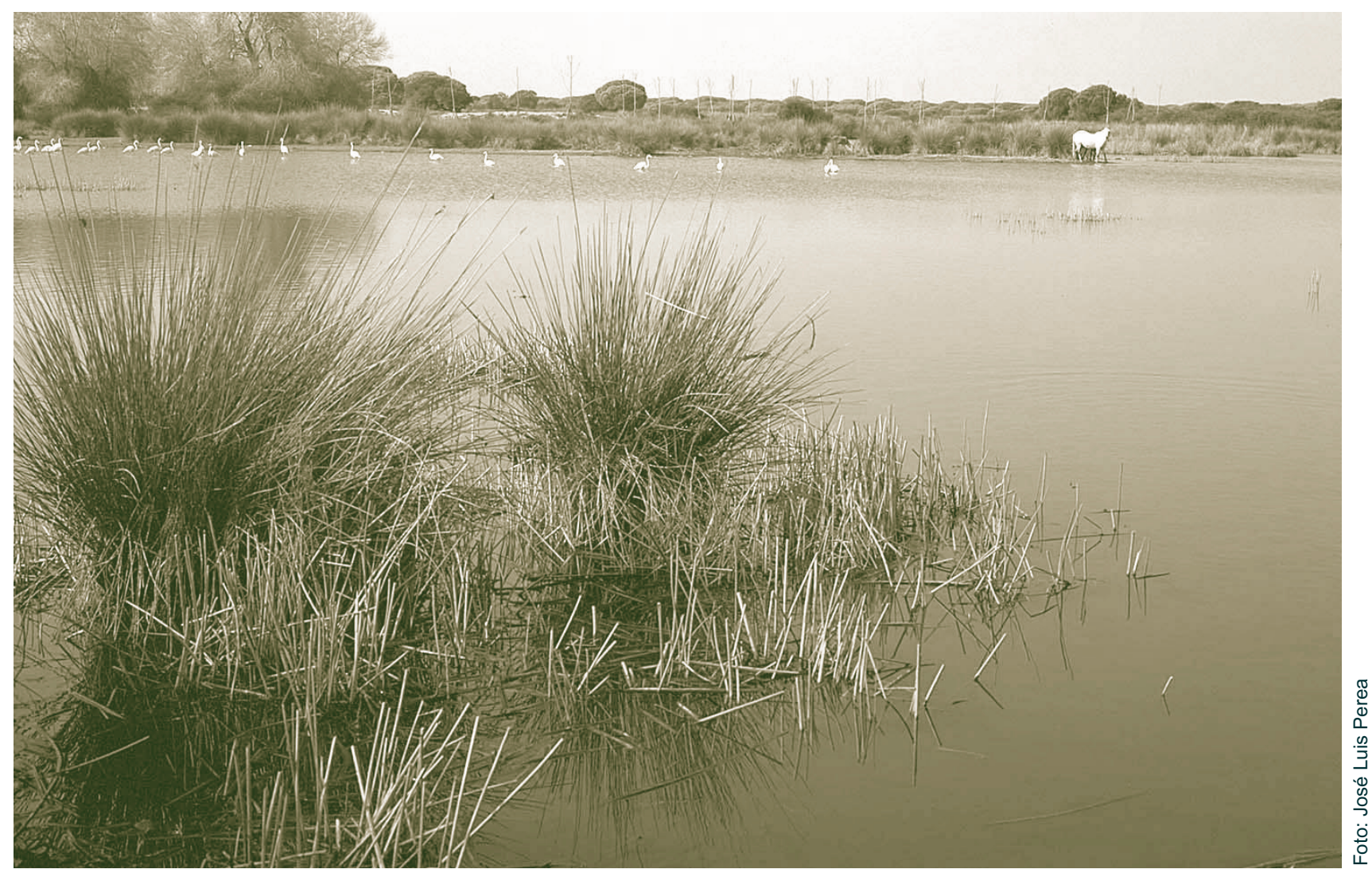

Los sensibles humedales de la Peninsula Ibérica.

\section{FORMACIÓN CONTINUA EMPRESA- RIAL}

Las exigencias de formación en las empresas se vinculan, cada vez en mayor medida, al conocimiento de la problemática ambiental, los sistemas de gestión medioambiental o las auditorías medioambientales. Las empresas demandan asimismo, dentro de la formación ambiental, conocimientos en el ámbito legislativo, de desarrollo de modelos de gestión y nuevas tecnologías, y estudios de eficiencia, gestión y protección de las empresas.

La Fundación Biodiversidad recoge esta preocupación por la protección y preservación de nuestro entorno natural, y como institución dedicada a la investigación, formación, sensibilización y gestión contribuye al desarrollo del empleo, impulsando el espíritu de empresa, la adaptabilidad, la igualdad de oportunidades y la inversión en recursos humanos.
Así, la Fundación Biodiversidad gestiona para el Estado Español, dentro del Programa Operativo "Iniciativa Empresarial y Formación Continua", cofinanciado por el Fondo Social Europeo, las actuaciones referentes a la formación en el sector medioambiental, la sensibilización de PYMES sobre aspectos ligados al medio ambiente y el análisis de necesidades formativas y la creación de estructuras. Entre los objetivos prioritarios destaca el desarrollo de actividades en materia de formación y sensibilización ambiental para trabajadores, empresarios y directivos de pequeñas y medianas empresas, autónomos y profesionales cuya actividad incida directa o indirectamente sobre el medio ambiente.

Las actuaciones están cofinanciadas por el Fondo Social Europeo - cuya misión es contribuir al desarrollo del empleo impulsando la captación de inserción profesional, el espíritu de empresa, la adaptabilidad, la igualdad de oportunidades y la inversión en recursos humanos-y la Fundación 


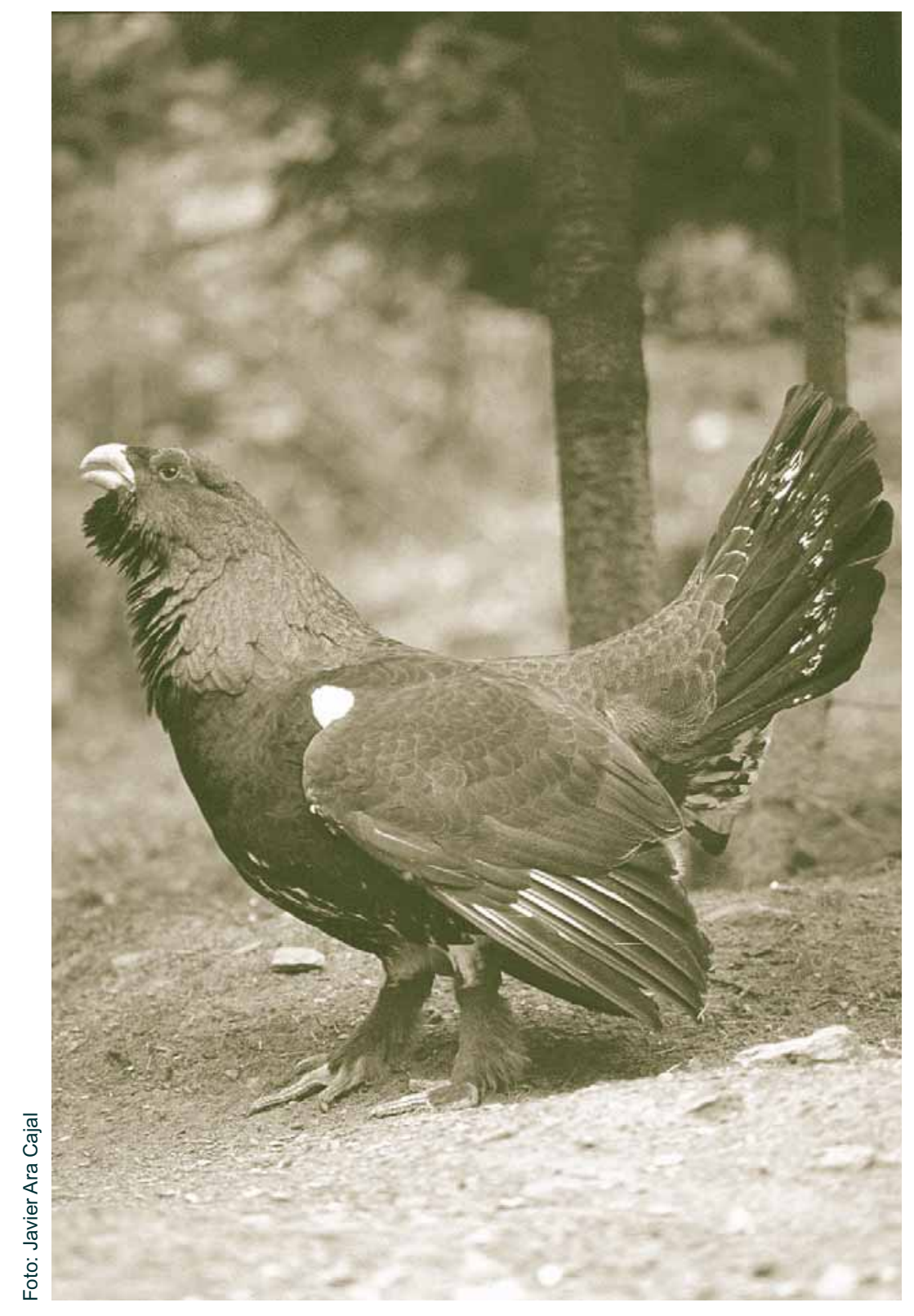

Ejemplar de urogallo cantando.

Biodiversidad. Su ámbito de actuación es nacional, engloba las regiones Objetivo 1 y 3 y tiene una vigencia hasta el 2006.

Las acciones objeto de cofinanciación son las de sensibilización, análisis de necesidades formativas y formación. Esta última se impartirá a varios niveles: formación básica, especializada y formación de educadores ambientales, mediante distintas modalidades que contemplan la presencial, a distancia o la mixta.

El objetivo prioritario del Programa Operativo consiste en asegurar la actualización del nivel de competencias de los trabajadores aumentando, a través de la formación continua, su capacidad para adaptarse a la evolución de las necesidades requeridas por el sistema productivo en un sector todavía incipiente para las empresas como es el del medio ambiente. Los destinatarios de las operaciones a realizar por la Fundación Biodiversidad en el marco de este Programa Operativo son los trabajadores de PYME, empresarios y profesionales, con especial atención a los colectivos de personas mayores de 45 años, mujeres, trabajadores de baja cualificación y discapacitados. 


\section{SENSIBILIZACIÓN AMBIENTAL}

Entre los objetivos de la Fundación Biodiversidad destacan promover la sensibilización y trasladar a los ciudadanos la conservación del medio ambiente. Para cumplir con ellos, la Fundación Biodiversidad impulsa, en colaboración con otros organismos e instituciones, proyectos para la protección de la flora y la fauna. En este sentido, ha iniciado, junto con la Universidad de Extremadura, la investigación para determinar la pureza genética del ciervo ibérico. Promueve la cultura del reciclaje entre los estudiantes más jóvenes a través del programa "Envases y Residuos de envases. Cerrando Ciclos", una iniciativa de educación y sensibilización ambiental y que consta de un material didáctico editado por APAS, Asociación para la Promoción de Actividades Socioculturales, en el que participan Ecoembes y Ecovidrio.

Asimismo, la Fundación Biodiversidad está realizando junto con la Federación Española de Caza, un estudio genético, sanitario y de comportamiento de la perdiz roja autóctona, para demostrar la impureza o ausencia de impureza en la perdiz roja. La sensibilización del sector turístico hacia el medio ambiente es otro de los proyectos que desarrolla en colaboración con el Instituto para la Calidad Turística Española, a través de la implantación de la Norma de Calidad Turística Ambiental Q Verde. Esta norma se adapta a todos los subsectores del negocio turístico que se involucren en la mejora de la calidad de sus productos y servicios y supone el respaldo institucional a la implantación de la Marca de Calidad Turística Española.

En colaboración con la FUCI, Federación de Usuarios-Consumidores Independientes, colabora en la puesta en marcha de actividades relacionadas con la convocatoria anual del concurso "Bandera Verde-Ciudad Sostenible", un premio dirigido a los ayuntamientos españoles que promueven el consumo responsable y que destacan por su gestión modélica de los residuos. Para orientar en este premio, ambas instituciones editan un manual de buenas prácticas que contiene recomendaciones y ex- periencias que animan a un consumo responsable con un desarrollo sostenible y colaboran en la actividad escolar "Detectives Ecológicos. Por una Ciudad Bandera", una acción de voluntariado juvenil mediante la cual se involucra a los estudiantes para que participen en el desarrollo de una cultura sostenible.

Asimismo, la Fundación Biodiversidad patrocina diversas exposiciones que fomentan la protección de la flora y la fauna. La delegación de Sevilla acoge el mes de mayo una exposición cuyo objetivo es la preservación del quebrantahuesos y que promueve la Fundación para la Conservación del Quebrantahuesos.

Para llevar a cabo estos proyectos de sensibilización y de promoción externa de sus actividades, la Fundación Biodiversidad cuenta con los departamentos de Comunicación y Relaciones Externas y el de Estudios y Publicaciones. El primero se encarga de dar a conocer a los ciudadanos y de difundir a los medios de comunicación y a los organismos interesados las actividades que ésta desarrolla. Asimismo, publicar, exponer, exportar y transmitir la imagen y los proyectos que gestiona, evalúa, realiza, y en los que participa la Fundación Biodiversidad.

Desde el área de Estudios y Publicaciones de la Fundación Biodiversidad se llevan a cabo estudios en el campo de la economía del medio ambiente, la fiscalidad del medio ambiente, permisos de emisión negociables y otros instrumentos económicos para la gestión medioambiental, energía y medio ambiente, y cambio climático, entre otros. Asimismo, ha creado la convocatoria de premios, tanto de investigación como de tesis doctorales, que estimulen el interés de los investigadores por los temas medioambientales y que, posteriormente, publicará la Fundación Biodiversidad. Este departamento pretende impulsar y realizar estudios en múltiples áreas de estudios medioambientales. En ambos casos, la Fundación Biodiversidad publicará estos estudios y serán expuestos en seminarios, congresos, jornadas y en todos aquellos foros que organice esta institución. 